\title{
Prediction of the outlet temperature of an experimental heat exchanger using artificial neural networks
}

\author{
A. Tlatelpa-Becerro ${ }^{1}$, L. Castro-Gómez ${ }^{1}$, G. Urquiza $^{1}$ \\ \& R. Rico-Martínez ${ }^{2}$ \\ ${ }^{1}$ Universidad Autonoma del Estado de Morelos-CIICAp, México \\ ${ }^{2}$ Departamento de Ingeniería Química, \\ Instituto Tecnológico de Celaya, México
}

\begin{abstract}
Artificial neural networks (ANNs) coupled with a Kalman filter were applied to predict the outlet temperatures of a laboratory crossflow heat exchanger. Time series of inlet temperatures were obtained and used as input values for training and testing the ANN. The ANN showed good prediction capabilities, but the high noise presented in the inlet variables frequently prevented the ANN prediction to match the evolution observed in the experiment. In order to further improve the tracking capabilities of the ANN, a Kalman filter was introduced. The results of the assisted scheme showed the maximum deviation between the predicted results and experimental data with relative errors below 0.007 y $0.002 \%$ for the water and air outlet temperature respectively. This assisted model reference strategy can be applied for thermal analysis in engineering applications and the development of predictive control applications in real time.

Keywords: artificial neural networks, time series, Kalman filter, heat exchanger.
\end{abstract}

\section{Introduction}

The heat exchangers are complex devices used in a wide variety of engineering applications, for example, refrigeration and air conditioning. The complexity of these systems is due to its geometric configuration and physical phenomena present in the heat transfer. Most simulations of heat exchangers and other components of thermal systems have focused on their steady state behavior for 
predicting the heat transfer rate required for the system design. However, physicochemical systems that are far from thermodynamic equilibrium may exhibit complex dynamical behaviors. The complex behaviors are related to their inherent nonlinear character due to several factors such as corrosion and fouling, leading to cavity erosion. However, the dynamic response of these devices is very important as they must are be controlled in some way, and it is therefore necessary to develop models capable of adapting to these occurrences. The purpose of this study is to demonstrate the capabilities of an assisted reference model strategy in order to predict crossflow heat exchanger outlet temperatures. The strategy is illustrated in an air-water heat exchanger using artificial neural networks as the reference model coupled with a Kalman filter in order to improve the tracking capability of the ANN.

This paper is organized as follow: section 2 contains a description of the experimental setup as well as the time series obtained. In section 3 neural network configuration and Kalman filter assisted strategy are described. In section 4 the prediction results with ANN and the Kalman filter are presented. Finally, in section 5 a brief discussion over the results and methodology is presented.

\section{Physical model and experimental data}

In Figure 1 the experimental apparatus can be observed. The heat exchanger has been locally designed by research group and built at Thermohydraulic Laboratory of the CIICAp-UAEM. There is a single water-side circuit which goes back and forth across the tubes of the heat exchanger. Type-T isolated thermocouples were used to measure the air inlet and outlet temperature, and for the water-side a flowmeter was used. The flow of air in the tunnel is due to a fan controlled by a variable speed drive operated manually. The air speed is measured using a digital anemometer, located upstream. The data acquisition board used can obtain measurements of up to 32 different channels, simultaneously. The data acquisition

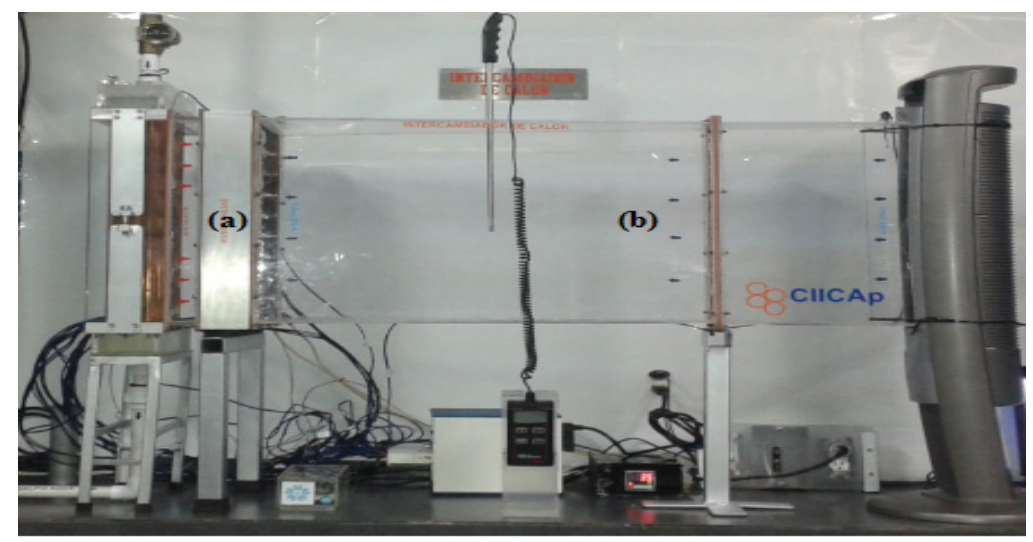

Figure 1: Experimental setup: (a) heat exchanger; (b) wind tunnel. 
board receives information about inlet and outlet temperatures from sides, air and water, the air speed and the time at which the measurements were taken. LabVIEW was used for experimental data acquisition. Time-dependent information regarding the air and water inlet temperatures, $T_{a}^{i n}$ and $T_{w}^{i n}$, respectively, and the air and water outlet temperatures, $T_{a}^{\text {out }}$ and $T_{w}^{\text {out }}$, respectively, were stored.

This system is known to exhibit a large variety of complex behaviors as the temperature varies. Time series processed were obtained from the experimental heat exchanger during a single test run. Figures 2 and 3 show the temperatures measurement versus time, which was obtained with 368 and 751 points per each time series for train and tests the ANN, respectively.
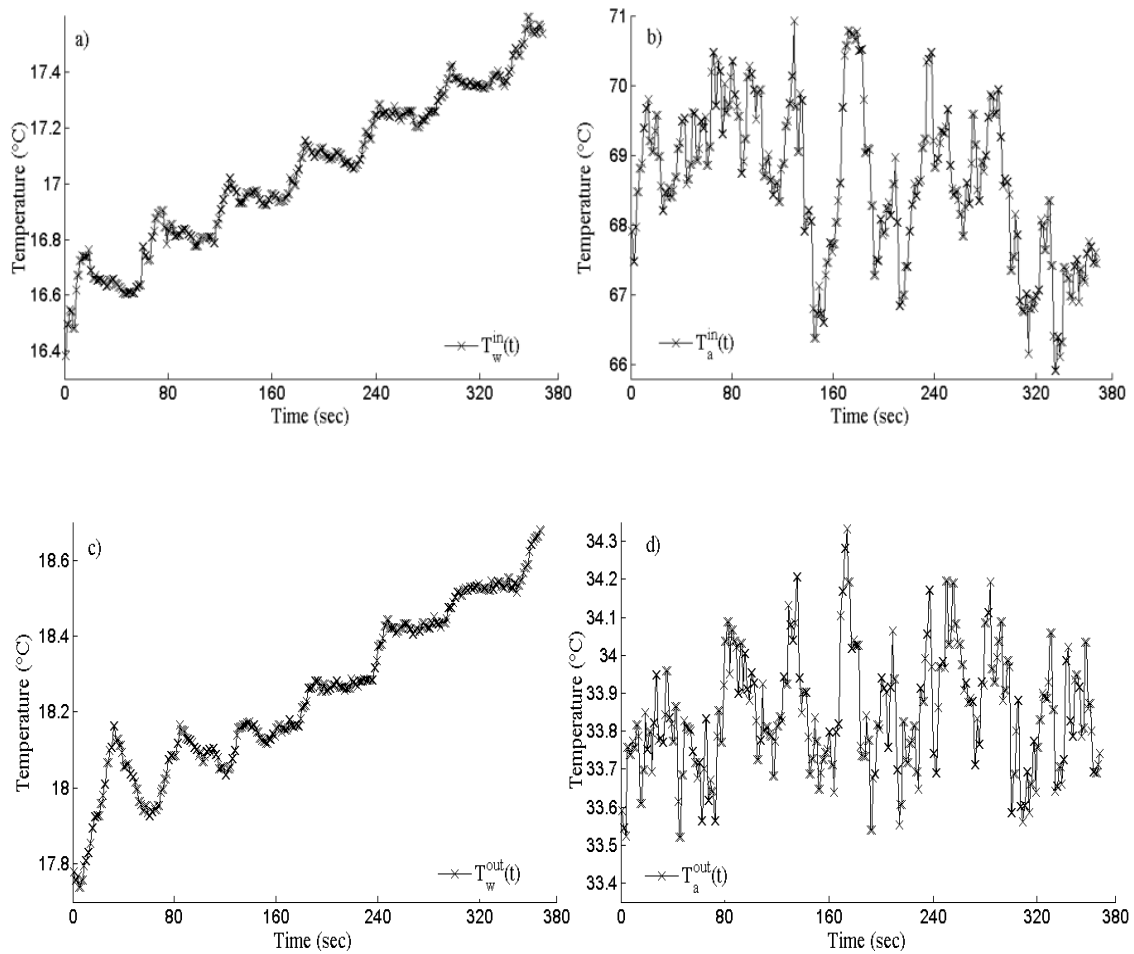

Figure 2: Experimental time series used to train the ANN: From left to right, (a) water inlet temperature, (b) air inlet temperature, (c) water outlet temperature, and (d) air outlet temperature.

Figure 3 shows the time series portion allocated to test the ANN. The temperatures, $T_{w}^{i n}(t)$ and $T_{a}^{i n}(t)$, were used as inputs to the ANN and thereby predict the outlet temperatures. 

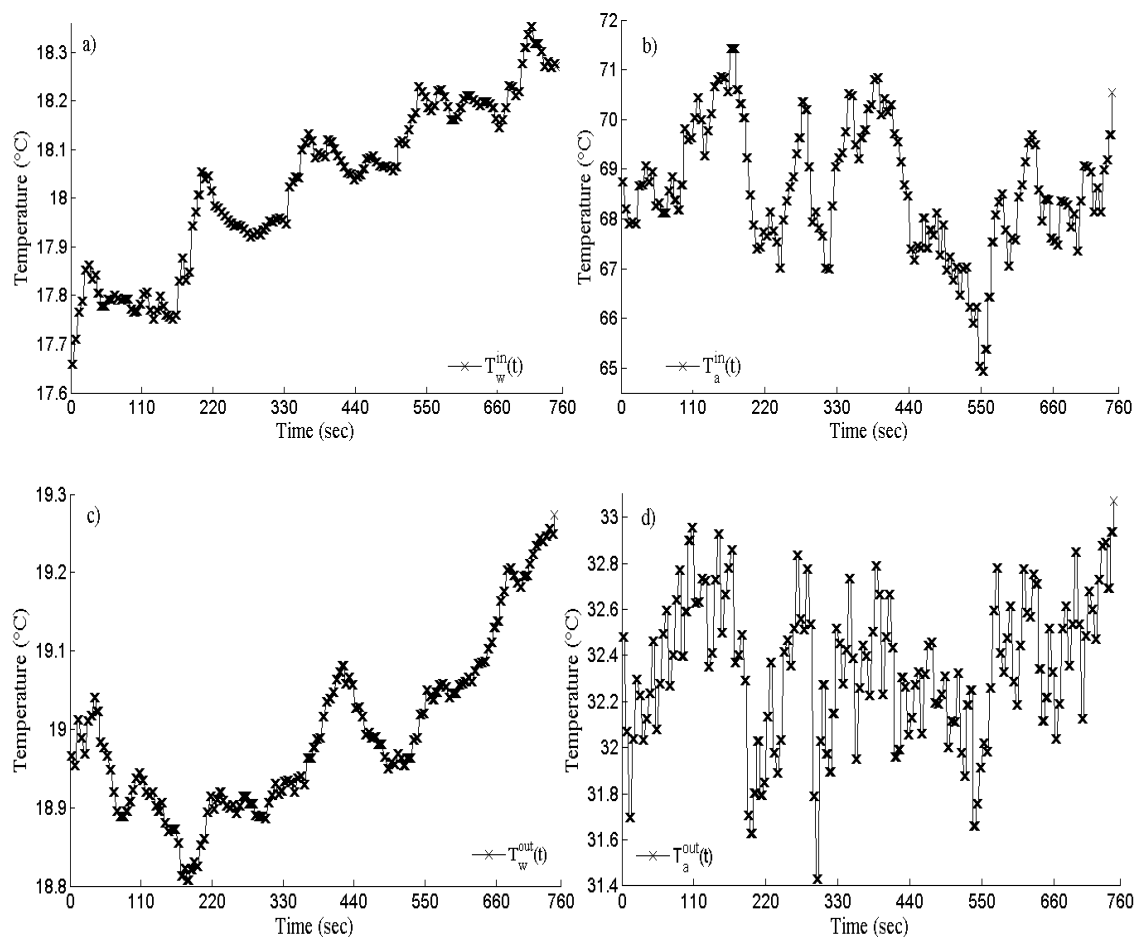

Figure 3: Experimental time series used to test the ANN: From left to right, (a) water inlet temperature, (b) air inlet temperature, (c) water outlet temperature, and (d) air outlet temperature.

\section{Neural network configuration and Kalman filter}

The ANNs by using a large number of parameters (weights and biases) can learn and recognize complicated non-linear functions. They are widely used in a variety of fields, among them, Rico-Martinez et al. [1] presented an ANN configuration which couples a nonlinear principal component network for data processing with a composite ANN based on a simple integrator scheme. Anderson et al. [2] have applied ANN for system identification and series time analysis. González-García et al. [3] developed a methodology for the identification of distributed parameter systems, based on artificial neural network architectures, motivated by standard numerical discretization techniques used for the solution of partial differential equations. The ANNs are defined by their input and output layer, plus a number of hidden layers each comprised of so-called neurons. In theory, an ANN with two hidden layers with the appropriate number of neurons is able to estimate approximately any type of non-linear function. The neurons in each layer are linked to the adjacent layers and the strength of this interconnected configuration is determined by the weights. The interconnected structure is conducive to a logical relationship between input and output parameters. The number of neurons 
for the input and output layers is equal to the input and output variables, respectively. Nevertheless, in the hidden layer different numbers of neurons can be employed and its number may prevent the appropriate optimization of the network. In the network, information is transferred passing through the connections of the neurons in the adjacent layers.

Here, a feed forward network with an algorithm training to predict the outlet temperatures was build using the neural networks toolbox of MATLAB (2010). The ANN architecture is a four layer regular networks with 3 neurons in the input layer (inlet temperatures plus a delayed value of the inlet water temperature), two hidden layer with 12 neurons each, and 2 neurons in the output layer as shown in Figure 4. As there is currently no rigorous way to determine an optimum number of neurons, the choice in the number of neurons in the hidden layers is somewhat arbitrary. Our choice here reflects a compromise between the computational effort required to train the $\mathrm{ANN}$ and an estimate of the minimum number of neurons needed to capture the underlying dynamics. This compromise is tested during the process of validation of the results: in this particular case the optimum number of neurons was determined based on the minimum value of MSE of the training and prediction sets. Using a Polak-Ribiere conjugate gradient algorithm and calculating the derivatives with respect to network parameters using the backpropagation algorithm the ANN was trained. The ANN with 12 neurons per hidden layer was found to yield qualitative correct results and quantitative results with a MSE below 0.014 .

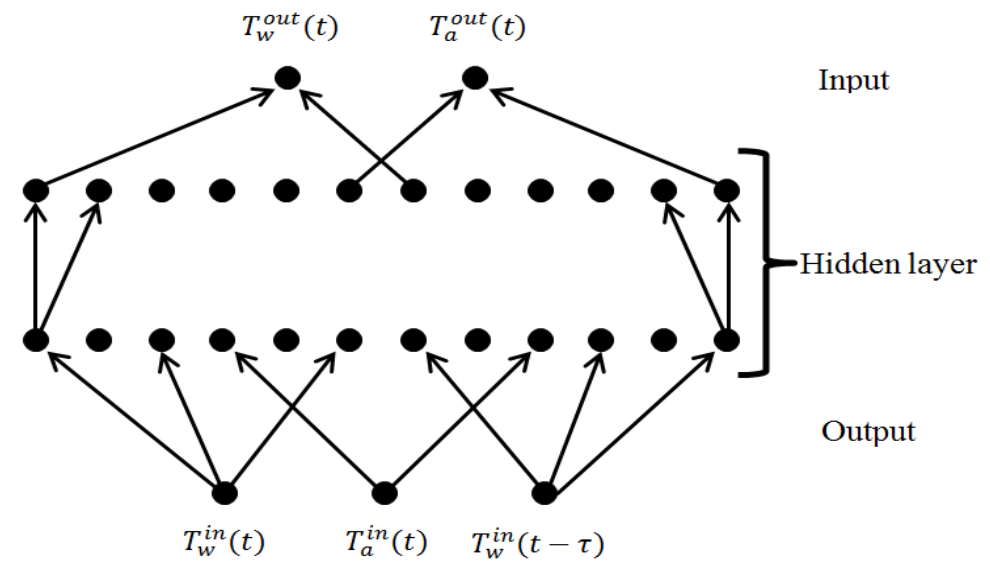

Figure 4: $\quad$ ANN architecture used. $T_{a}^{i n}(t), T_{w}^{i n}(t)$ and $T_{w}^{i n}(t-\tau)$ are the inputs to be ANN which represent the state of the system. $T_{a}^{\text {out }}(\mathrm{t})$ and $T_{w}^{\text {out }}(t)$ are the prediction of the ANN. Only a few connections of the fully interconnected neurons are depicted.

The architecture ANN was formed by three vectors at inlet: $T_{a}^{i n}(t)$ and $T_{w}^{i n}(t)$ at the current time, and a time delayed measurement for water temperature $T_{w}^{i n}(t-$ $\tau$ ). While, $T_{a}^{\text {out }}(\mathrm{t})$ and $T_{w}^{\text {out }}(t)$ at the current time (as shown in Figure 4) were the 
desired output targets. The input and output neurons of the ANN used are linear, while the neurons in the hidden layer are nonlinear with activation tangent sigmoid function (tanhsig). Since this activation function gives values between 0 and 1 , the network inputs and target values were accordingly normalized [4].

In order to improve the prediction of the output temperatures in the presence of slowly varying components (i.e. changes in the heat exchanger performance due to fouling in its tube walls), a hybrid strategy via incorporation of a modified Kalman filter was proposed. In this sense, through the Kalman filter online adaptation capabilities, one provides a mechanism for compensating modelling errors, improving the robustness of the neural network predictions. The Kalman filter is a numerical tool composed of dynamic equations that have been widely used for stochastic estimation and filtering of noisy measurements. It is an optimum filter in the sense that it minimizes the estimated covariance of the error in experimental measurements. This strategy has been previously described by Ramírez-Álvarez et al. [5, 6] for other nonlinear estimation tasks.

\section{Results}

Artificial neural networks (ANNs) were applied to predict the experimental heat exchanger outlet temperatures. The ANN by itself showed good prediction capabilities, but the high noise presented in the inlet variables frequently prevented the ANN prediction to match the evolution observed in the experiment for the water and air temperatures, as shown in Figures 5 and 6.

In order to further improve the tracking capabilities of the ANN, a modified Kalman filter was introduced. The Kalman filter is a modified version that takes into account the nonlinearity of the system and is intended to assist in compensating this deviation. With the application the Kalman filter the tracking capabilities were improved. The results are shown also in Figures 5 and 6.

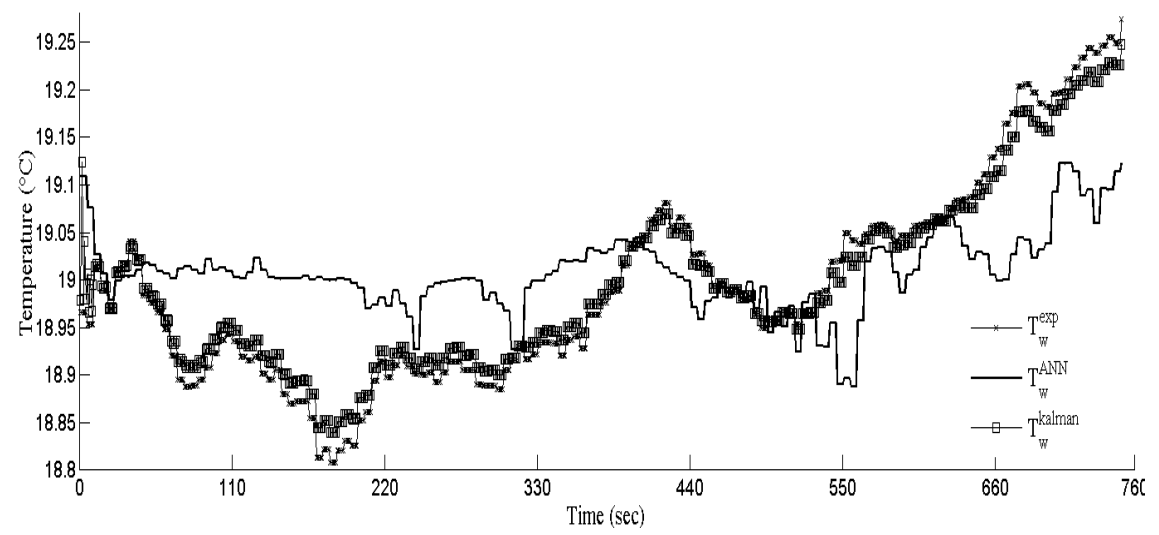

Figure 5: Comparison of the water temperature: $T_{w}^{\exp }$ Experimental temperature. $T_{w}^{A N N}$ ANN prediction. $T_{w}^{\text {Kalman }}$ and ANN assisted by the Kalman filter. 


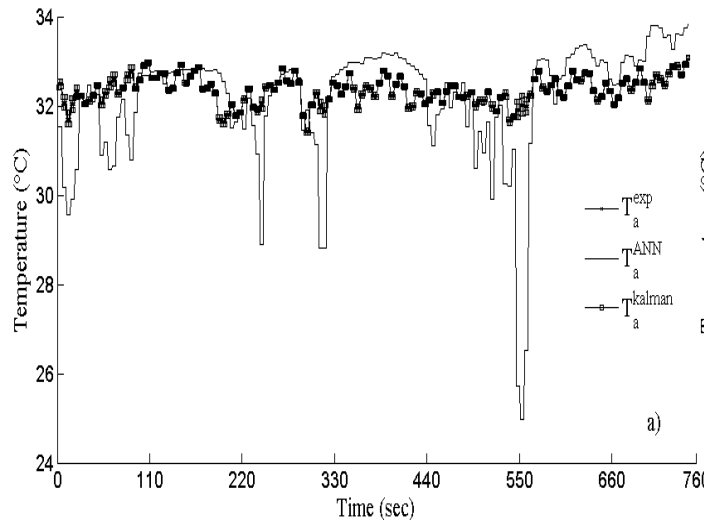

(a)

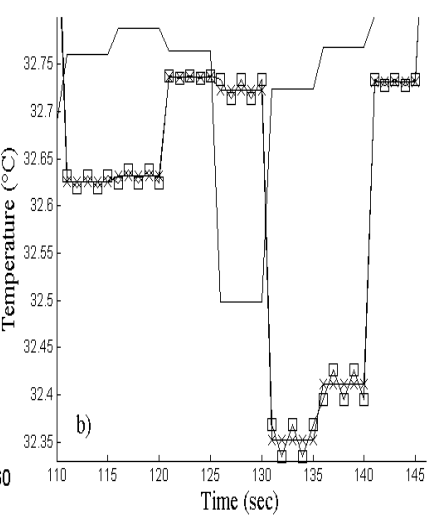

(b)

Figure 6: (a) Comparison of the air temperature: $T_{a}^{\text {exp }}$ Experimental temperature. $T_{a}^{A N N}$ ANN prediction $T_{a}^{\text {Kalman }}$ and ANN assisted by the Kalman filter. (b) Close-up on a time-series portion in order to better observe the deviations.

The results of the assisted scheme showed the maximum deviation between the predicted results and experimental data with relative errors below 0.02 y $0.25 \%$ for the water and air outlet temperature respectively only with the ANN. When the ANN is coupled with the modified Kalman filter one observe relative errors below 0.002 and $0.007 \%$ for the water and air outlet temperature respectively, as show in Figure 7.

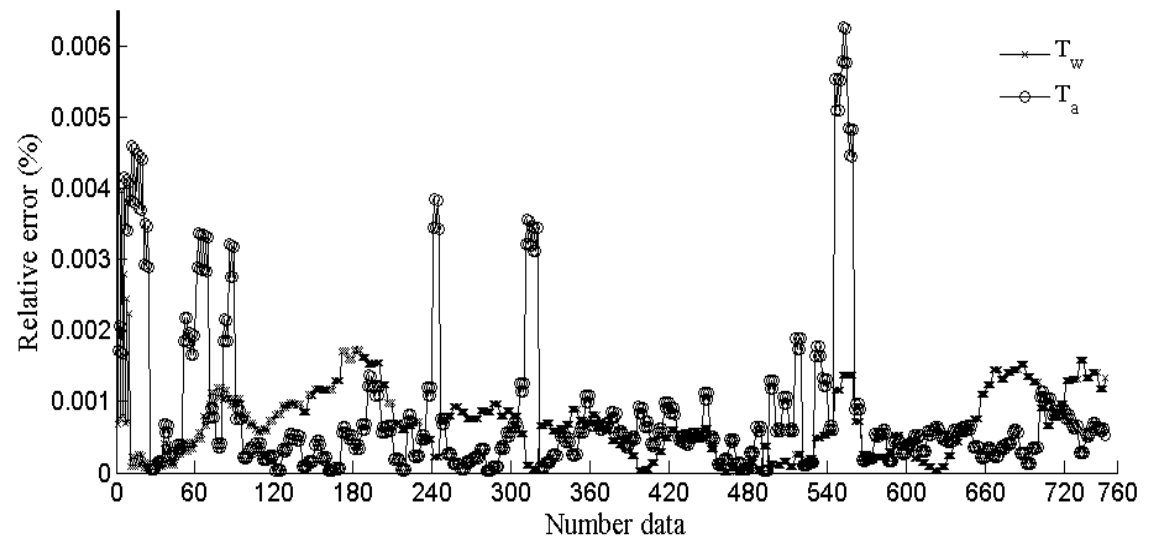

Figure 7: Relative errors obtained with the Kalman filter: $T_{w}$ water temperature. $T_{a}$ air temperature. 


\section{Conclusions}

Main goal was to develop an algorithm to closely predict the outlet temperature of a laboratory crossflow heat exchanger. Time series were obtained and processed to build the predictive model based on the ANN. Using a predictive artificial neural network (ANN) approximation coupled to a modified Kalman filter such task has been accomplished.

The assisted scheme showed good results greatly reducing the average deviation observed by the prediction of the ANN by itself, and properly handling the high-noise signal observed for the inlet temperatures.

The proposed model reference strategy can be applied for thermal analysis in engineering applications and the development of predictive control applications in real time. Currently, we are developing such real-time applications.

\section{References}

[1] Rico-Martínez R., Krischer K. and Kevrekidis I.G., Discrete vs continuoustime nonlinear signal processing of $\mathrm{Cu}$ electrodissolution data. Chemical Engineering Communications, 118 (1), pp. 25-48, 1992.

[2] Anderson J.S., Kevrekidis I.G. and Rico-Martínez R., A comparison of recurrent training algorithms for time series analysis and system identification. Computer chem. Engn. 20 (S), pp. 751-756, 1996.

[3] González-García R., Rico-Martínez R. and Kevrekidis I.G., Identification of distributed parameter systems: A neural net based approach. Computer chem. Engn. 22 (Suppl), pp. 965-968, 1998.

[4] Khataee A.R., Kasiri M.B.., Artificial neural networks modeling of contaminated water treatment processes by homogeneous and heterogeneous nanocatalysis. Journal of Molecular Catalysis A: Chemical. 331(1), pp. 86$100,2010$.

[5] Ramírez-Álvarez E., González-Figueredo C. and Rico-Martínez R., Models assisted by nonlinear Kalman filters as a strategy for real-time bifurcation detection. 20th European symposium on computer aided process engineering - ESCAPE20, Editors. S. Pierucci and G. Buzzi Ferraris, Elsevier: B.V., 2010.

[6] Ramírez-Álvarez E., Calderón Ramírez M., Rico-Martínez R., GonzálezFigueredo C. and Parmananda P., Detecting bifurcations in an electrochemical cell employing an assisted reference model strategy. The Journal of Physical Chemistry. 117, pp. 535-540, 2013. 